\title{
Temperature effects on light yield and pulse shape discrimination capability of siloxane based scintillators
}

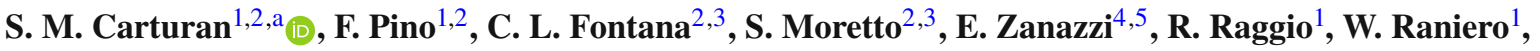 \\ A. Quaranta ${ }^{4,5}$ \\ ${ }^{1}$ INFN, Laboratori Nazionali di Legnaro, Viale dell’Università 2, 35020 Legnaro, Padua, Italy \\ 2 Department of Physics and Astronomy "Galilei”, University of Padova, Via Marzolo 8, 35100 Padua, Italy \\ ${ }^{3}$ INFN-Sezione di Padova, Via Marzolo 8, 35100 Padua, Italy \\ ${ }^{4}$ Department of Industrial Engineering, University of Trento, Via Sommarive 9, 38123 Povo, Trento, Italy \\ ${ }^{5}$ INFN-Trento Institute of Fundamental Physics and Applications (TIFPA), Via Sommarive 14, 38123 Povo, Trento, Italy
}

Received: 21 September 2020 / Accepted: 3 November 2020 / Published online: 17 November 2020

(C) The Author(s) 2020

\begin{abstract}
In this work, we report the study of temperature effects on light yield of siloxane-based scintillators and on $n / \gamma$ discrimination capability, in terms of pulse shape discrimination (PSD). The solid scintillators are composed of phenyl containing polysiloxane (PMPS100), as a base polymer, loaded with moderate amounts $(6 \mathrm{wt} \%)$ of 2,5 diphenyloxazole (PPO) as a primary dye and Lumogen Violet $(\mathrm{LV})$ as waveshifter. The samples were heated in the range of $60-150{ }^{\circ} \mathrm{C}$ and scintillation performance were tested both after annealing for $24 \mathrm{~h}$ and in real time during heating. Light yield of siloxane-based scintillators containing $6 \mathrm{wt} \% \mathrm{PPO}$ heated at $100^{\circ} \mathrm{C}$ is very close to the room-temperature value, while heating at $120^{\circ} \mathrm{C}$ causes a decrease of light yield (LY) of $17 \%$. In addition, the figure of merit (FoM) for $\mathrm{n} / \gamma$ discrimination of the scintillator shows a sensible worsening of the discrimination performances in case of prolonged treatment at $120^{\circ} \mathrm{C}$. Similar tests are made using the commercial plastic scintillator EJ-299 (currently named EJ-276), based on polyvinyltoluene (PVT). In this case, the light yield undergoes a much more rapid deterioration with annealing temperature, and at $70{ }^{\circ} \mathrm{C}$ it is reduced to $60 \%$ of the original value. The discrimination capability of EJ-299 decreases upon heating at $70^{\circ} \mathrm{C}$ as well, with a $20 \%$ reduction of FoM; meanwhile for $\mathrm{T}>70^{\circ} \mathrm{C}$ the mechanical and optical features are remarkably degraded. The mass loss of primary dye PPO from the siloxane scintillator as a function of treatment temperature and initial dye concentration has been evaluated and compared to the behaviour of EJ-299. This measurement allows to single out and characterize a series of processes occurring during heating, which are relevant to the whole performance of the system under study, such as sublimation at the interface, thermally induced photooxidation of components,
\end{abstract}

a e-mail: carturan@lnl.infn.it (corresponding author) diffusion of fluorophores from the polymer bulk to the surface. The variation in luminescence characteristics have been analyzed by excitation/fluorescence spectroscopy and timeresolved fluorescence spectroscopy, in order to correlate the annealing treatment with the primary dye loss by sublimation, formation of superficial aggregates and/or degradation of the scintillator components in the synthesized siloxane scintillator.

\section{Introduction}

Polymer-based scintillators are used for several applications: radiation detectors in calorimeters to detect and track muons $[1,2]$, reliable and cost-effective radiation portal monitors [3], as alpha/beta counters in the radioactivity soil scanning [4] and surveillance of radiation-exposed workers [5]. They bear preferential features versus inorganic scintillators, such as ease of manufacture at various thickness and shape, reduced costs, negligible sensitivity to the environment, lightweight and fast response time [6].

Siloxane-based scintillators have been studied as for composition, optical properties, scintillation light output from several decades [7-10]; in comparison with ordinary plastic scintillators, they demonstrated effective light yield, detection of $\gamma$-rays, alpha particles and fast neutrons. Moreover, loaded with suitable boron-containing compounds, they preserved transparency and showed good thermal neutron sensitivity [11]. The detection of thermal neutrons has also been pursued with remarkably high efficiency using polysiloxane as a supporting matrix for $\mathrm{ZnS}: \mathrm{Ag}$ powder (EJ-600) and ${ }^{6} \mathrm{LiF}$ nanocrystal synthesized by co-precipitation $[12,13]$. Very recently, siloxane-based sensors showed optimal dis- 
crimination between fast neutrons and $\gamma$-rays by exploiting the pulse shape analysis of the scintillation light [14]. This extremely attractive feature is reached by using polymethylphenylsiloxane (PMPS) embedding moderate amounts (2$8 \%$ ) of 2,5-diphenyloxazole (PPO) collected in phenyl rich sites, thus promoting the close proximity for triplet-triplet annihilation and the related delayed fluorescence. These systems were extensively studied by optical spectroscopy and scintillation analyses.

The first commercial plastic scintillator for the discrimination of fast neutrons and gamma rays, formerly filed under the name of EJ-299, is currently known as EJ-276 [15]. This material displays optimal PSD capabilities, reaching performances comparable with the ones of liquid scintillators, known for high toxicity and safety concerns. The base polymer matrix, polyvinyltoluene (PVT), is a glassy polymer $(\mathrm{Tg}$ $\left.>90{ }^{\circ} \mathrm{C}\right)$ with moderate thermal resistance $\left(\mathrm{Td} \approx 100^{\circ} \mathrm{C}\right)$, but the high loading of primary dye, envisaged by previously published data on this material [16], has been shown to induce drastic deterioration of the base polymer features, such as glass transition, mechanical hardness and dimensional stability over time [17-19]. In the case of PVT based traditional scintillator, i.e. EJ-200 of Eljen Technology and BC-404 of Saint-Gobain, that contains a low amount of primary dye $(<$ $2 \% \mathrm{wt}$ ), heating up to $50{ }^{\circ} \mathrm{C}$ has little influence on the light yield [20].

Physical instability, softness and dye aggregation over long-term operation have been recently greatly improved by Zaitseva et al., acting on the cross-linking density of the base polymer [21].

Very recently, Lim et al. [22] exploited a commercial formulation of high-index siloxane to prepare efficient scintillators doped with a moderate quantity of PPO thus further supporting the outcome reported in Ref. [14]. The scintillators described therein even exceed the performance of commercial plastic scintillators both in light yield and PSD capability, thus demonstrating that the features of siloxane-based detectors might be still enhanced.

Siloxanes are very popular as heat and radiation resistant materials, as witnessed by their successful application in different fields spanning from cookware to aerospace components. From this point of view, siloxane scintillators may be regarded as benchmark sensors in those environments where not only PSD is demanded but also long-term resistance at high temperature with capability preservation.

In this work, we deepen the behaviour of siloxane-based scintillators both after and during heating at different temperatures, ranging from 60 to $150^{\circ} \mathrm{C}$. Optical and scintillation properties are monitored as a function of temperature and are compared with EJ-299 scintillator properties under the same operational conditions. This work confirms the reliability of siloxane scintillators also at temperatures which are typically out of reach for standard plastic scintillators.

\section{Experimental}

The scintillators pellets were prepared starting from the homopolymer polymethyl-phenylsiloxane vinyl terminated (PMPS) added with suitable amounts of co-methylhydrophenylmethylsiloxane hydride terminated as cross-linker and Pt based Karstedt catalyst. This resin, composed of the base resin with $100 \%$ Si-phenyl unit in the macromolecule, was chosen owing to the demonstrated very good PSD performance [14]. All reagents were purchased from Gelest, USA.

The viscous mixture was doped with PPO (scintillation grade, Acros Organics) and Lumogen Violet (LV, BASF), according to selected formulations, prior to vulcanization. This process was carried out in an oven at $60{ }^{\circ} \mathrm{C}$ for $24 \mathrm{~h}$ and afforded cylindrical samples with $2 \mathrm{~cm}$ diameter and 1 $\mathrm{cm}$ height. To investigate the effect of heating on the optical properties of both base matrix and primary dye (PPO), one series of samples was produced without adding the secondary dye (LV). Moreover, the mass loss of PMPS based samples containing only PPO at different concentrations has been estimated at different annealing temperatures, using a Gibertini balance with accuracy $0.0001 \mathrm{~g}$.

Photoluminescence properties of samples, as they are after preparation and after annealing in the air for $24 \mathrm{~h}$ in the range from 80 to $150{ }^{\circ} \mathrm{C}\left(60^{\circ} \mathrm{C}\right.$ corresponds to the polymerization temperature), were measured in front face geometry using a Jasco FP-6300 spectrofluorimeter, equipped with a $150 \mathrm{~W}$ Xe lamp; the spectra were collected at room temperature. With the same approach, transmittance spectra were collected using a Jasco spectrophotometer V-570 from samples $1 \mathrm{~mm}$ thick and from samples of EJ-299 with the same thickness. Luminescence lifetime measurements were performed with a PicoQuant acquisition module (PicoHarp 300), exciting the samples with $285 \mathrm{~nm}$ pulsed LED (Horiba N-280, pulse width $1.2 \mathrm{~ns}$ ) and recording the emission intensity at 400 and $450 \mathrm{~nm}$ with a monochromator (Horiba, f/4, 1200 lines $/ \mathrm{mm}, 8 \mathrm{~nm}$ of bandwidth) equipped with a PMT (Hamamatsu $\mathrm{H} 10721-04$, rise time $0.57 \mathrm{~ns}$ ).

Scintillators with PPO $2 \%$ and $6 \%$ wt. were produced, fixing the amount of $\mathrm{LV}$ at $0.02 \% \mathrm{wt}$. In previous works, these concentrations gave good light yield and $\mathrm{n} / \gamma$ pulse shape discrimination capability [14]. To assess their resistance to overheating, a specific set-up was assembled (Fig. 1), where both a schematic drawing (Fig. 1a) and a photo (Fig. 1c) are shown. For the mass loss analyses and for the study on the PPO luminescence properties change as a function of the temperature we produced samples containing only PPO at concentrations of $1 \%, 2 \%, 4 \%$ and $6 \%$ wt.

The experimental set-up was made up of the sample coupled to the PMT (Hamamatsu H6524) through an acrylic light guide ( $2 \mathrm{~cm}$ diameter, $5 \mathrm{~cm}$ length). Good optical matching was obtained with silicone grease (Dow, Dowsil Optical couplant Q2-3067). The heating element (flexible silicone heater 

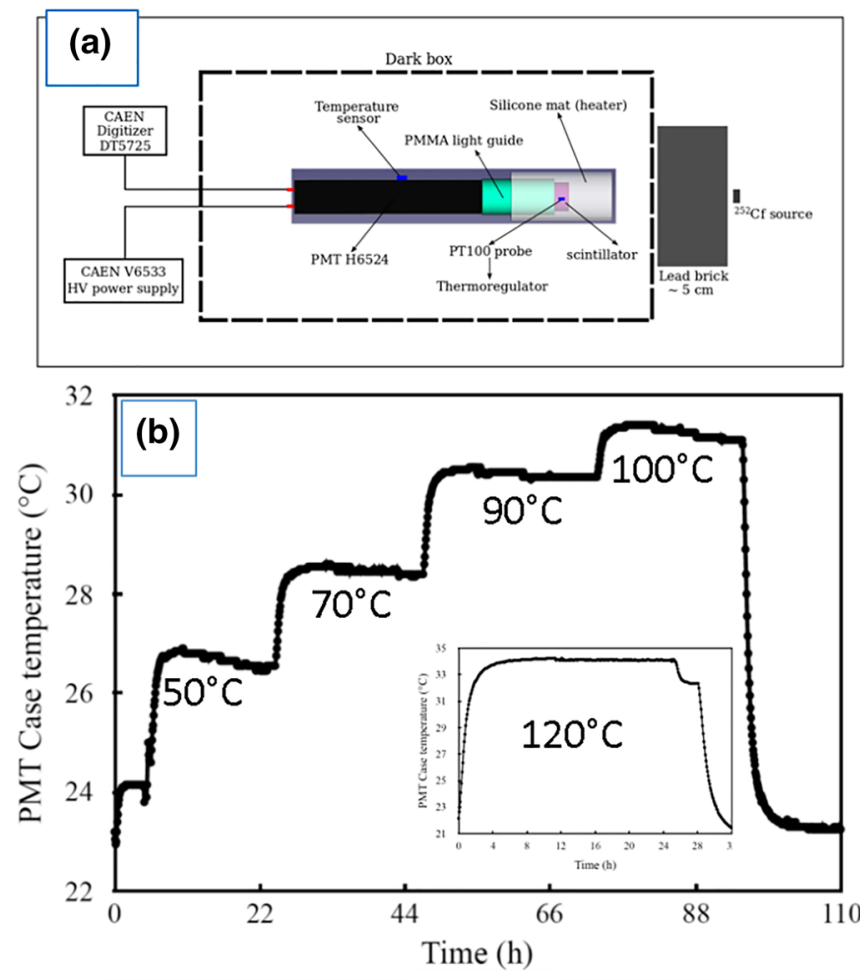

Fig. 1 a Scheme of the set-up used to measure the light output with calibrated sources and the $\mathrm{n} / \gamma$ bi-dimensional plot of the scintillators at different heating temperatures; $\mathbf{b}$ temperature recorded on PMT side

mat, RS components) was used to wrap the light guide coupled on one end to the PMT (Hamamatsu H6524) glass window and on the other end to the scintillator pellet.

During scintillation measurements, the temperature of the sample is set and controlled by the heating element connected to a thermoregulator (Gefran-1000) equipped with a PT100 resistance thermometer, allowing to reach the set temperature with an accuracy of $2{ }^{\circ} \mathrm{C}$. The use of the light guide is needed to preserve the PMT from overheating during operation, and a K-type thermocouple was set in close contact with the junction interface PMT glass/light guide to check the thermal conditions of the PMT during the acquisition time. The poly (methyl methacrylate) light guide material has a high decomposition temperature (around $330^{\circ} \mathrm{C}$ ) and its glass transition temperature is about $110^{\circ} \mathrm{C}$ [23], thus it can be used without relevant decrease in optical and mechanical properties up to $120^{\circ} \mathrm{C}$. The heating rate cannot be controlled by the regulator, but it was manually set to avoid heat spikes on the sample at a rate of around $5{ }^{\circ} \mathrm{C} / \mathrm{min}$. Figure $1 \mathrm{~b}$ reports the temperature reached by the PMT during the heating steps of the sample. The maximum reached temperature is about 34 ${ }^{\circ} \mathrm{C}$, thus well below the limit of operation indicated by the manufacturer $\left(50^{\circ} \mathrm{C}\right)$.

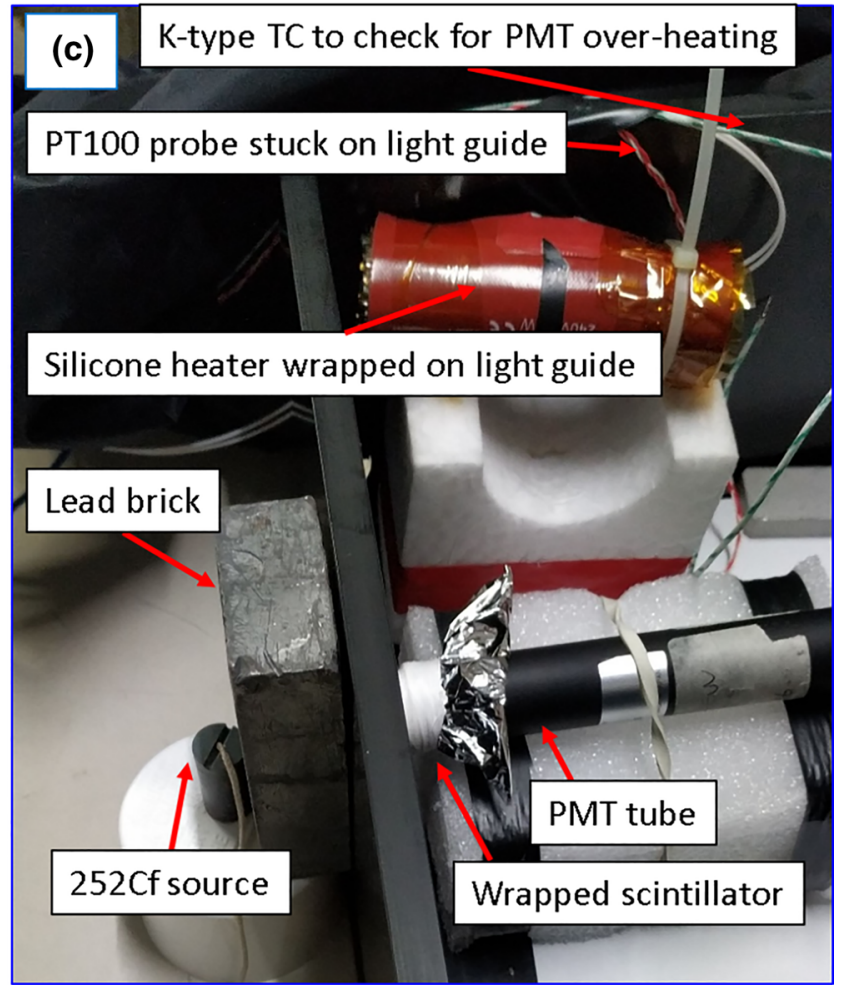

during the heating steps of the sample at the temperatures reported in the graph. The step at $120^{\circ} \mathrm{C}$ was reached by a separate test. $\mathbf{c}$ photo of the set-up

The scintillation yield was estimated using ${ }^{137} \mathrm{Cs} \gamma$ source with the scintillator coupled to a light guide whose opposite side is facing the PMT: this allows to measure the light output as compared to a standard scintillator of the same size (EJ299, Eljen Technology). At the same time, the expression of results as a ratio against the commercial standard allows to neglect the contribution of PMT gain dependence with temperature, which will be the same for both our sample and the standard. Each source was placed front-facing the scintillator, at a distance of $\sim 10 \mathrm{~cm}$. Scintillation spectra were collected for $10 \mathrm{~min}$, once the thermal equilibrium was reached.

The pulse shape discrimination (PSD) features of the scintillators were studied using a ${ }^{252} \mathrm{Cf}$ source (neutron emission rate about $5 \times 10^{4} \mathrm{~ns}$ ) placed at about $10 \mathrm{~cm}$ in front of the scintillator. Each measurement lasted $20 \mathrm{~h}$ approximately. Thereafter, measurements with ${ }^{137} \mathrm{Cs}$ were performed. PMT signals were acquired with a CAEN DT5725 fast digitizer (14 bit ADC resolution, 250 Msamples/s). Open source software [24] was used for the data acquisition [25]. On-line digital pulse processing was performed by the signal digitizer, which allows saving for each event: the timestamp, the partial integral, the total integral and, optionally, the complete waveform. The double gate integration method was applied 
to perform the pulse shape analysis, as described in [13], and the pulse shape parameter (PSP) was computed as:

$P S P=\frac{Q_{\text {tail }}}{Q_{\text {total }}}$,

where $\mathrm{Q}_{\text {total }}$ and $\mathrm{Q}_{\text {tail }}$ are, respectively, the total and the tail integrals of the pulse. Widths of the integration gates were optimized by analyzing the data acquired with unheated scintillators [14]. In order to characterize the $\mathrm{n} / \gamma$ discrimination capability, we used the figure of merit (FoM) obtained by analyzing the PSP distributions defined as in [13]

$F o M=\Delta /\left(\delta_{\mathrm{n}}+\delta_{\gamma}\right)$,

where $\Delta$ is the distance between the centroids of neutron and $\gamma$ signal distributions and $\delta_{\mathrm{n}}+\delta_{\gamma}$ is the sum of their FWHMs.

\section{Results}

\subsection{Weight loss after annealing}

Samples with different amount of PPO have been weighed before and after thermal treatment, and the percentage mass loss values are reported in Table 1 . The values obtained for EJ-299 are also reported for comparison.

The trend shows a linear relationship between the mass loss and the annealing temperature. This finding is a clear indication of the occurrence of two processes: sublimation of PPO from the surface and diffusion of PPO through the polymer towards the sample surface. As the PPO is released from the surface with a rate depending on the selected temperature, the diffusion of PPO from the bulk to the surface compensates the loss, thus leading to the linear correlation. The fact that mass loss is due to PPO evaporation and not to the polymer decomposition is demonstrated by the starshaped point in Fig. 2a, where the pure PMPS siloxane without PPO is treated at the maximum temperature used in this experiment and negligible mass variation has been observed. It is worth to notice that PMPS with 6\% PPO loses half the content of PPO after treatment at $150{ }^{\circ} \mathrm{C}$.

By analyzing the problem of the evaporation from the surface of a semi-infinite solid containing a starting concentration $\mathrm{c}_{0}$, we obtain that the total amount $\mathrm{M}$, expressed in material quantity per area unit, of molecules leaving the solid at a time $t$ is given by [26]:

$M=2 c_{0} \sqrt{\frac{D t}{\pi}}$,

where $D$ is the diffusion coefficient of the molecule in the solid and $t$ is the total annealing time expressed in seconds.

In order to exploit the relationship, we express the lost amount of PPO, $M$, in $\mathrm{mg} / \mathrm{cm}^{2}$, taking into account the cylindrical sample area and the concentration $\mathrm{c}_{0}$ in $\mathrm{mg} / \mathrm{cm}^{3}$.
Table 1 Results of weight loss measurements on PMPS samples with different PPO concentration treated at selected temperatures

\begin{tabular}{llll}
\hline Label & PPO wt $\%$ & $\mathrm{~T}\left({ }^{\circ} \mathrm{C}\right)$ & Mass loss \% \\
\hline PMPS & 0 & 150 & $0.29 \pm 0.02$ \\
EJ-299 & 30 & 80 & $0.42 \pm 0.02$ \\
& & 100 & $1.95 \pm 0.02$ \\
& 120 & $3.90 \pm 0.02$ \\
PPO1 & 1 & 150 & $7.08 \pm 0.02$ \\
& & 80 & $0.01 \pm 0.02$ \\
& & 100 & $0.19 \pm 0.02$ \\
PPO2 & 120 & $0.27 \pm 0.01$ \\
& 2 & 150 & $1.11 \pm 0.01$ \\
& & 80 & $0.091 \pm 0.009$ \\
PPO4 & & 100 & $0.31 \pm 0.01$ \\
& 4 & 120 & $0.47 \pm 0.01$ \\
& & 80 & $1.46 \pm 0.01$ \\
& & 100 & $0.22 \pm 0.01$ \\
& & 120 & $0.59 \pm 0.01$ \\
& & 150 & $1.13 \pm 0.01$ \\
& 6 & 150 & $2.23 \pm 0.01$ \\
& & 120 & $0.15 \pm 0.02$ \\
& & 150 & $0.57 \pm 0.01$ \\
& & & $2.89 \pm 0.01$ \\
& & 150.01 \\
\hline
\end{tabular}

${ }^{\mathrm{a}}$ The error is estimated applying the propagation theory on the basis of the balance accuracy

By plotting $M$ as a function of $\mathrm{c}_{0}$ (Fig. 2b) we obtain a line whose slope is equal to $2 \sqrt{D t / \pi}$.

$M=a c_{0}+b$.

Therefore, we can calculate the value of PPO diffusion coefficient at different temperatures.

The PPO concentration in the samples produced in this work is known, whereas the PPO concentration in EJ-299 is assumed to be $30 \mathrm{wt} \%$ [16].

By using the treatment time $t$ in seconds, we obtain for each treatment temperature the diffusion coefficient in $\mathrm{cm}^{2} / \mathrm{s}$ and the standard deviation following the equations:

$$
\begin{gathered}
D=\frac{a^{2} \pi}{t}, \\
\sigma_{D}=\frac{2 a \pi}{t} \sigma_{a},
\end{gathered}
$$

where $\sigma_{\mathrm{a}}$ is the standard deviation of the angular coefficient from the linear fit. The estimated values for the diffusion coefficient are reported in Fig. 2b.

Interestingly, the EJ-299 displays a much lower mass loss than the one expected on the basis of the behaviour shown by PMPS samples. This can be deduced by observing the dashed lines of Fig. 2b, representing the linear fit of the experimental 


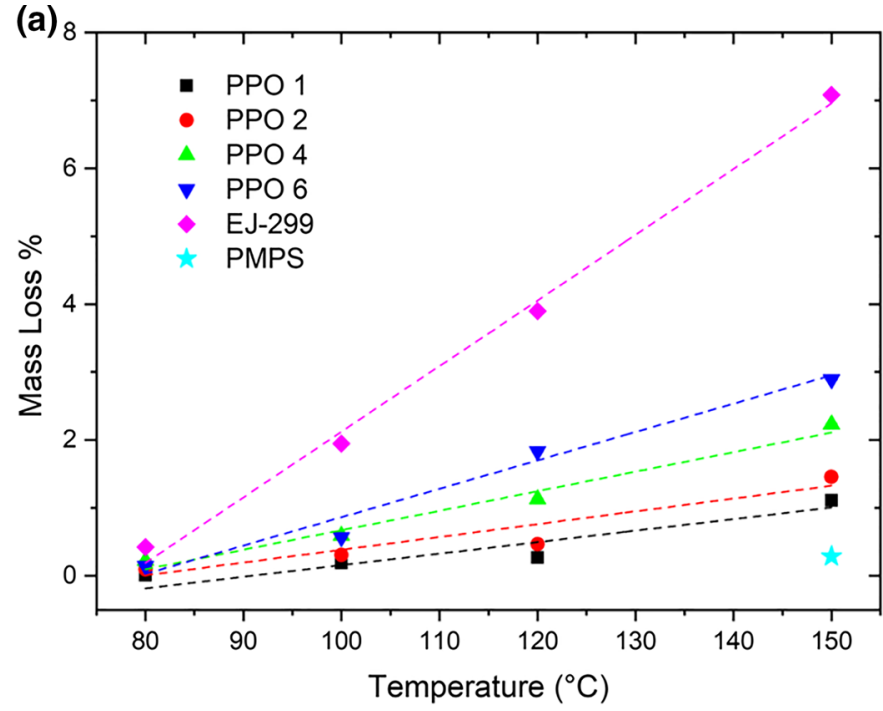

Fig. 2 a Mass loss \% of PMPS based samples containing different amount of PPO, the standard EJ-299 and the bare PMPS treated at different temperatures for $24 \mathrm{~h}$; dashed lines represent the result of linear fit for each data set. b PPO mass loss versus starting PPO concentration,

data limited to PMPS and extrapolated for high PPO concentration values. The EJ-299 related data lie well below the ones expected from an extrapolation of PMPS related data, therefore a much lower diffusion coefficient of PPO within the polymer matrix of EJ-299 can be envisaged.

\subsection{Optical transparency after annealing}

After heat treatment for $24 \mathrm{~h}$ at different temperatures, the samples $1 \mathrm{~mm}$ thick with increasing amount of PPO and the standard EJ-299 with the same thickness appeared as shown in the photo of Fig. 3a.

All the samples appear optically transparent up to $120^{\circ} \mathrm{C}$. Siloxane-based samples with PPO amount $<4 \%$ maintain their transparency up to $150^{\circ} \mathrm{C}$, whereas samples with 4 and $6 \%$ PPO show a moderate yellowing. The polyvinyltoluene based commercial scintillator EJ-299 displays evident yellowing after treatment at $150{ }^{\circ} \mathrm{C}$ and loss of mechanical resistance under compression (Fig. 3a, b). The faint yellowing of PMPS samples is ascribed to PPO thermal oxidation, whereas degradation of the polysiloxane base matrix is ruled out, owing to the well-demonstrated thermal resistance of silicones in general and, in particular, of phenyl-substituted ones [27]. As for the commercial scintillator EJ-299, thermal annealing at $150{ }^{\circ} \mathrm{C}$ for $24 \mathrm{~h}$ leads to intense yellow color, owing to thermally induced degradation of PPO, which is added in remarkably higher amount in EJ-299 than in polysiloxane, as mentioned before [21]. The carbon-based polymeric matrix, i.e. polyvinyltoluene [21], is not expected to undergo thermal degradation up to around $170{ }^{\circ} \mathrm{C}$ [28].

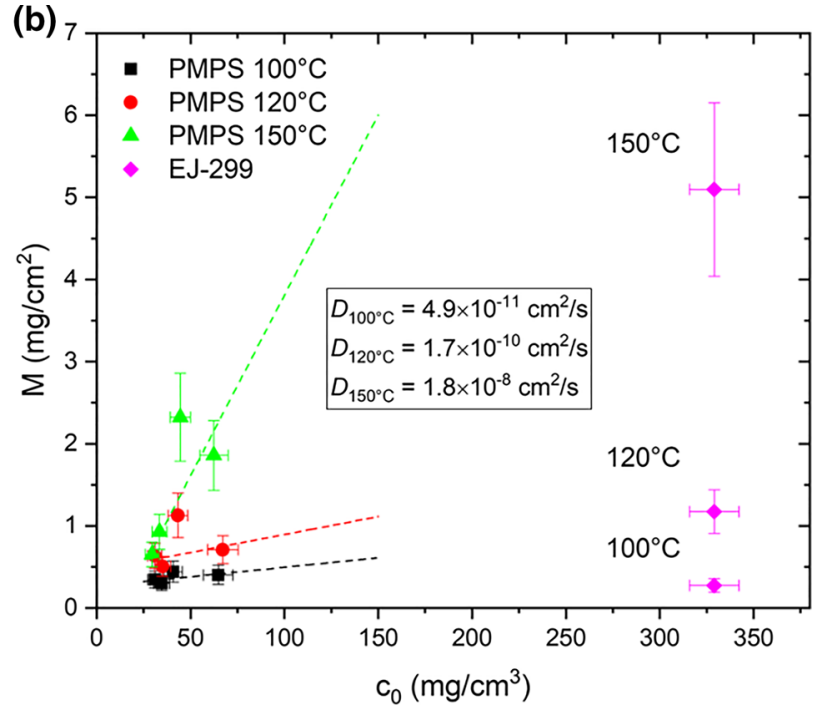

plotted for each annealing temperature. Dashed lines are the linear fit of data collected for PMPS based samples, whereas the EJ-299 data are left out of the fitting process

On the other hand, the glass transition temperature $\mathrm{T}_{\mathrm{g}}$ of the plastic base is very low, as a result of high PPO amount acting as a plasticizer (lower than $40{ }^{\circ} \mathrm{C}$ [19]), thus it softens and shows changes in shape after heating above $100{ }^{\circ} \mathrm{C}$ and successive cooling.

By observing the optical transmittance spectra in Fig. 3c, one relevant information is that the plateau of maximum optical transmission for PMPS samples is always lower than the standard EJ-299 scintillator and the optical transmittance in the range $400-800 \mathrm{~nm}$ decreases as PPO concentration increases from 1 up to $6 \%$. It is worth to remember that here the analyzed PMPS samples do not contain LV as wavelength shifter, hence the absorption edge is at around 375 $\mathrm{nm}$, whereas EJ-299 displays the edge at about $420 \mathrm{~nm}$.

The sample with 6\% PPO has a 50\% transmittance in the visible range, pointing to the presence of small aggregates that cause opacity due to light scattering. The heating step at $150{ }^{\circ} \mathrm{C}$ leads to a negligible decrease of transmittance in PMPS samples with the low amount of PPO, while the 6\% PPO sample shows reduced transmittance at around $380 \mathrm{~nm}$, corresponding to the absorption edge, and a slight blue-shift of the edge. The feature at $380 \mathrm{~nm}$ can be ascribed to the thermal oxidation of PPO with generation of colour centres, and the same process accounts for the loss of transmittance in heat treated EJ-299, which contains a higher amount of PPO. The small shift in the absorption edge of PMPS $6 \%$ PPO towards lower wavelength can be related to a decrease of PPO concentrations at the sample surface, as evidenced by the weight loss measurements. 


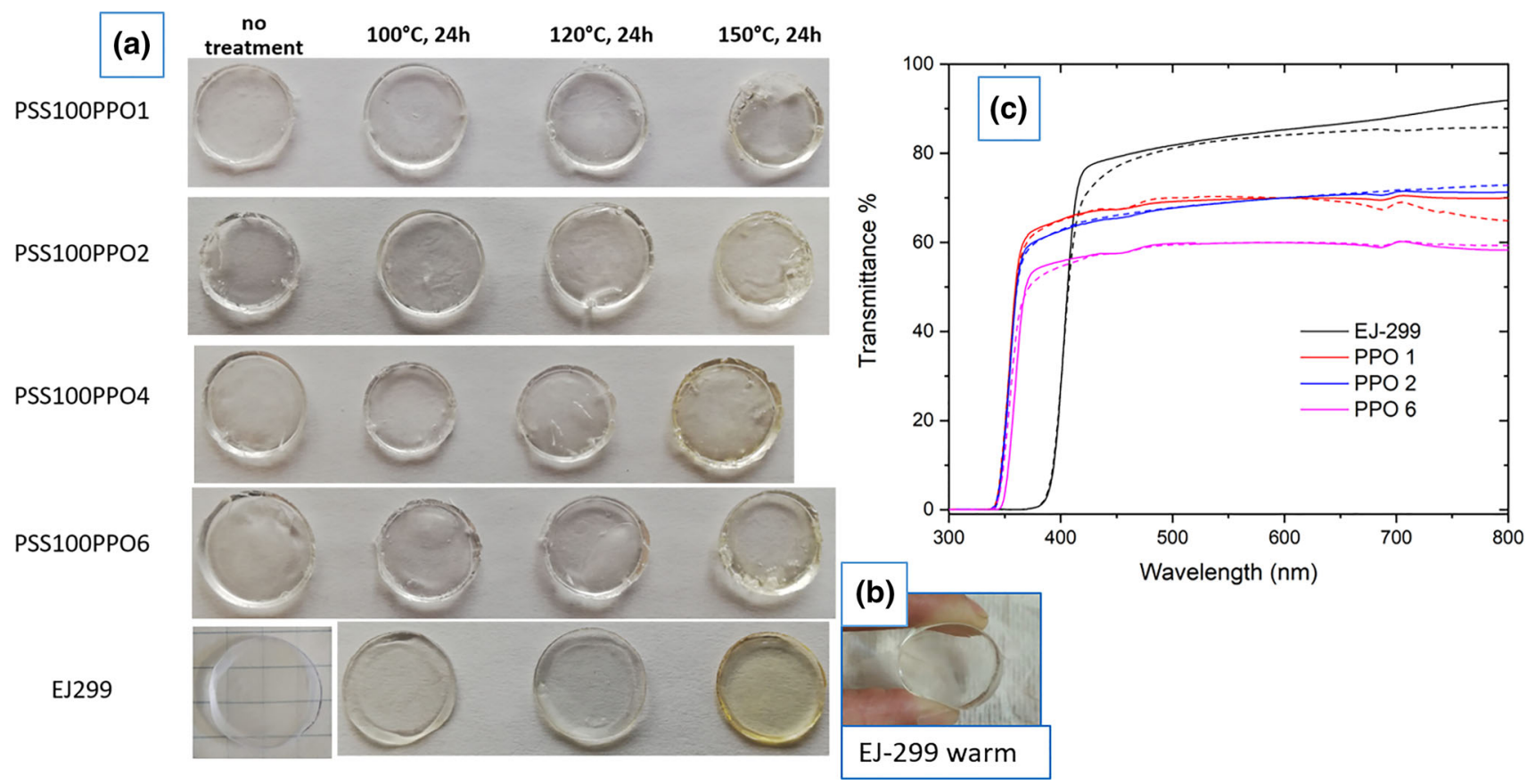

Fig. 3 a Images of the siloxane scintillators and the commercial one EJ-299 after annealing at different temperatures; b photo of the still warm EJ-299 after heating at $100{ }^{\circ} \mathrm{C}$ during manual compression; c
UV-Vis transmittance spectra of EJ-299 and siloxane with $2 \%$ and $6 \%$ PPO collected before (solid line) and after treatment at $150{ }^{\circ} \mathrm{C}$ (dashed line)
Samples with 2\% and 6\% PPO have been analyzed by luminescence spectroscopy, exciting the sample in front-face geometry. In Fig. 4, the normalized emission spectra collected using $\lambda_{\text {exc }}=280 \mathrm{~nm}$ are shown, after reticulation at $60{ }^{\circ} \mathrm{C}$ and after annealing at 100 and $150{ }^{\circ} \mathrm{C}$ for $24 \mathrm{~h}$.

The typical features of PPO are visible: excitation of the monomer form gives rise to a structured emission with a shoulder at $350 \mathrm{~nm}$ and the most intense vibronic peaks at 370 and $380 \mathrm{~nm}$, in agreement with the literature [29]. At high concentrations PPO is known to form excited dimeric species, i.e. excimers, characterized by emission features at higher wavelengths, contributing with an increase of the relative intensity in the higher wavelength tail of the dye spectrum. According to literature data, the excimer component appears in the range $440-450 \mathrm{~nm}$ and its intensity increases as the PPO concentration increases [29-32]. In liquid systems, the formation of excimers is a diffusion-controlled mechanism, depending on solvent parameters and temperature. In solid systems, the formation of PPO excimers is less explored; it has been recently studied by our group in polysiloxanes with PSD capability [14].

In the samples studied in the present work, a slightly higher relative intensity around $450 \mathrm{~nm}$ in samples with $6 \% \mathrm{PPO}$ (Fig. 4d) with respect to samples with $2 \%$ PPO, is indicative of the formation of excimer states [14]. As for the spectral shape, the treatment temperature does not remarkably affect the optical emission features for the PPO concentration range in PMPS considered in the present work. As for luminescence intensity variations, the evaluation is uncertain since the front-face analysis at $280 \mathrm{~nm}$ inspects very thin layers of the surface, leading to variations due to irregular surface morphologies and concentration distributions. As for the standard EJ-299, a visible change in spectral shape is observed at 150 ${ }^{\circ} \mathrm{C}$ in the spectra reported in the inset of Fig. 4a.

By comparing the normalized spectra of $2 \%$ and $6 \%$ samples treated at increasing temperature, it can be seen that the excimer component visible as a shoulder at $450 \mathrm{~nm}$ gradually decreases and at $150{ }^{\circ} \mathrm{C}$ the spectra of 2 and $6 \%$ are almost overlapped.

It is known that the presence of excimers can be more accurately studied through lifetime measurements. In Fig. 5, the time response of the luminescence at $450 \mathrm{~nm}$ of samples with 2 and 6\% PPO is reported.

Each decay profile has been fitted with a bi-exponential function according to the following equation

$I(t)=A_{f} e^{-t / \tau_{f}}+A_{s} e^{-t / \tau_{s}}$,

where $\tau_{\mathrm{f}}$ and $\tau_{\mathrm{s}}$ are respectively the lifetimes of the fast and slow component and $A_{f}$ and $A_{s}$ their amplitudes.

The fractional amplitude contribution of fast and slow components is calculated as follows [33]:

$f_{\text {fast }}=\frac{A_{\text {fast }} \tau_{\text {fast }}}{A_{\text {fast }} \tau_{\text {fast }}+A_{\text {slow }} \tau_{\text {slow }}}$. 

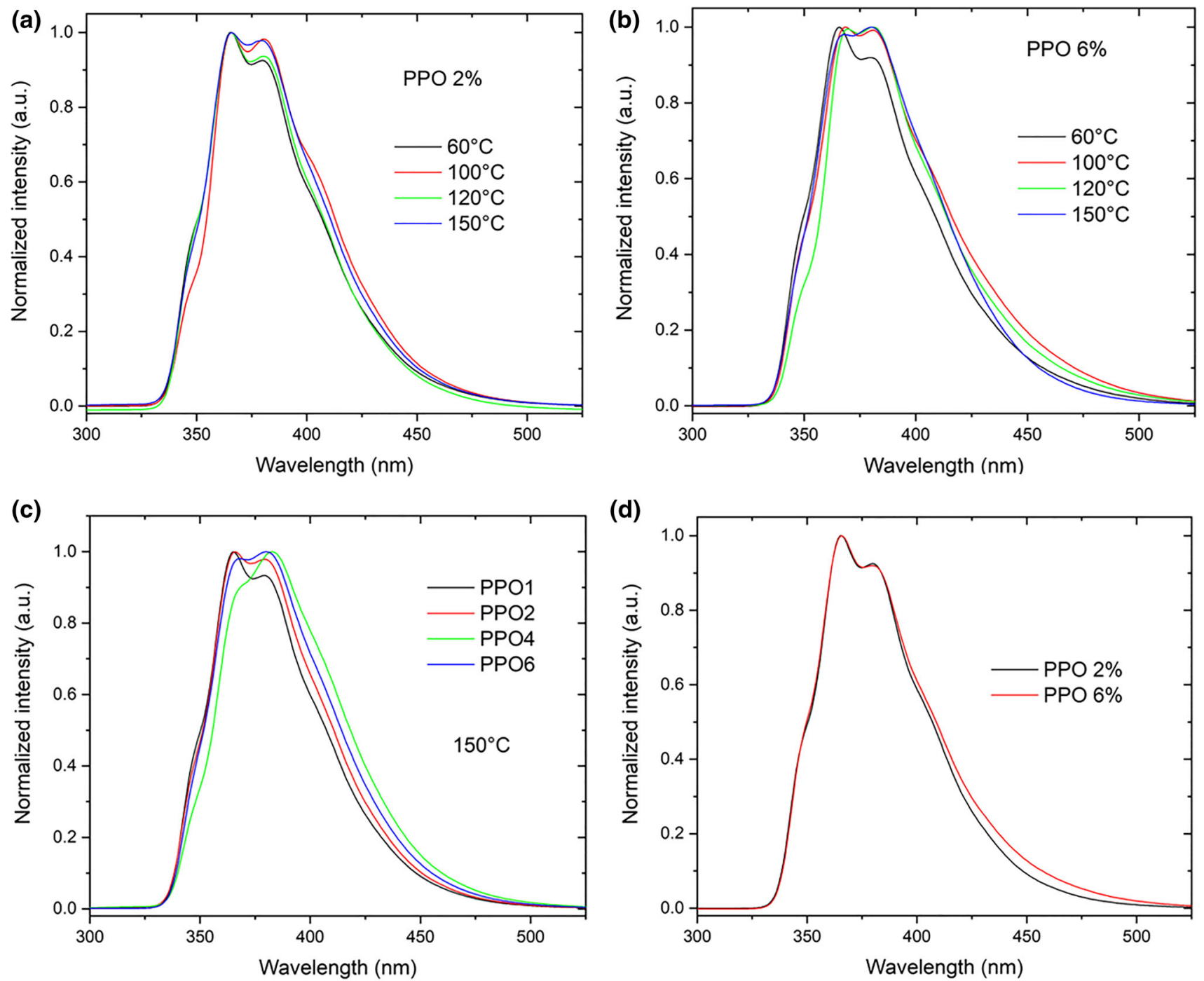

Fig. 4 Normalized fluorescence spectra collected $\mathbf{a}$ for samples of PMPS with 2\% PPO and b 6\% PPO after annealing at different temperatures, $\mathbf{c}$ for samples with increasing PPO treated at $150{ }^{\circ} \mathrm{C}$, $\mathbf{d}$ for untreated PMPS samples with 2 and $6 \%$ PPO. All the spectra are recorded using $\lambda$ ex 280 $\mathrm{nm}$
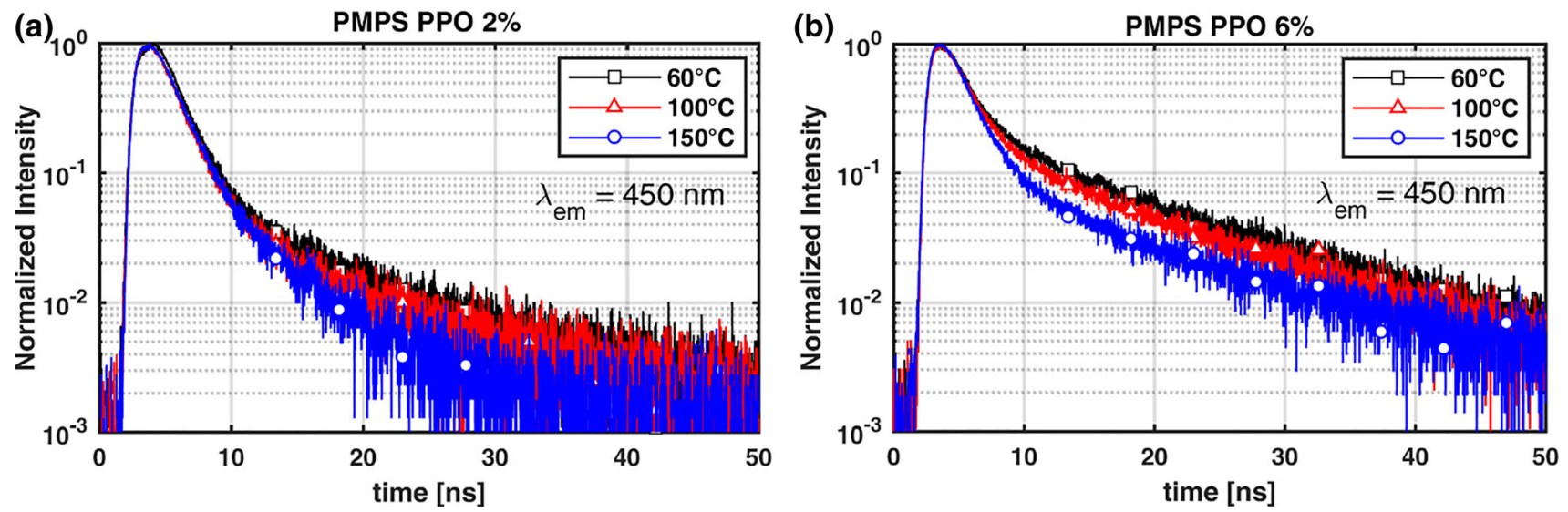

Fig. 5 Decay profiles of the fluorescence signal collected at $450 \mathrm{~nm}$ for the scintillators with $2 \%$ PPO (a) and $6 \%$ PPO (b) for different heating temperatures 


$$
f_{\text {slow }}=\frac{A_{\text {slow }} \tau_{\text {slow }}}{A_{\text {fast }} \tau_{\text {fast }}+A_{\text {slow }} \tau_{\text {slow }}} .
$$

In Table 1, the lifetimes and the ratios between the fractional contributions of fast and slow components for the different treatment temperatures and compositions are reported. It is worth to observe that the large error on $\tau_{\mathrm{s}}$ for the $2 \%$ PPO sample is due to the minimal contribution of this component, as can be seen in Table 2. This is in agreement with the negligible formation of long-living excimers previously suggested on the basis of the emission spectrum shape in Fig. $4 \mathrm{~d}$ and supported by previous literature work of Steingraber [34] and Yguerabide [35]. The effect of annealing temperature is clearly evident by observing the values of fast/slow components ratio: in the case of PPO $2 \%$, where the decay is dominated by the monomer species, the ratio is almost unaffected by heating, whereas a remarkable effect is clearly seen on the long tail of the signal for the case of $6 \% \mathrm{PPO}$, whose contribution decreases with the increase of the treatment temperature.

\subsection{Scintillation measurements}

The light output of scintillators at different temperatures was estimated by comparing the position of the Compton edges, in the pulse height spectra acquired with the ${ }^{137} \mathrm{Cs}$ source. The procedure used to find the position of the Compton edges is the same than the one applied in [13]. Results are given respect to the light output exhibited by the standard EJ-299 at room temperature. In Fig. 6a-c are shown the ${ }^{137} \mathrm{Cs}$ pulse height spectra corresponding to the standard EJ-299 and the PMPS based scintillators with $2 \%$ and $6 \%$ PPO at different temperatures. Figure $6 \mathrm{~d}$ shows the estimated light output, respect to EJ-299 at room temperature, taking into account the analysis of ${ }^{137} \mathrm{Cs}$ spectra.

It is worth to note that EJ-299 displays a remarkable decrease in light yield as the temperature is raised and the sample PMPS with 2\% PPO displays light loss as well, though the rate of scintillation degradation is lower than for EJ-299. In the case of EJ-299 at $70{ }^{\circ} \mathrm{C}$, the yield is almost halved as compared to the room temperature measured value, while the PMPS based scintillator with 6\% PPO still keeps the light yield almost constant irrespectively of the heating temperature up to $100^{\circ} \mathrm{C}$, showing a visible decrease only at $120^{\circ} \mathrm{C}$. It is worth to observe that PMT sensitivity variations caused by the temperature changes are expected to be lower (less than 3\%) than variations due to scintillation properties of the samples.

\subsection{Pulse shape discrimination features}

The widths of the integration gates were optimized in order to maximize the $n / \gamma$ discrimination performance. Figure 7 shows bi-dimensional histograms of the pulse shape discrimination parameter $\left(\mathrm{Q}_{\text {tail }} / \mathrm{Q}_{\text {total }}\right)$ versus the total integral $\left(\mathrm{Q}_{\text {total }}\right.$, quantity proportional to the total light output). The plots show the results obtained with the standard EJ-299 and with our PMPS based scintillator at room temperature $\left(\sim 25^{\circ} \mathrm{C}\right), 70$, 100 and $120^{\circ} \mathrm{C}$.

Figure 8a shows the $\mathrm{Q}_{\text {tail }} / \mathrm{Q}_{\text {total }}$ histograms corresponding to the EJ-299 and to the 6\% PPO PMPS scintillator, at room temperature and at $100{ }^{\circ} \mathrm{C}$. From data given in Fig. 7, only events with light output in the range $(480 \pm 75) \mathrm{keVee}$ were used to compute the figure of merit (FoM), using the same procedure applied in [13]. From these distributions, the FoM values were obtained for both types of scintillators at different temperatures (see Fig. 8b). As can be observed, FoM for EJ299 decreases with the temperature, with a plateau between $70^{\circ} \mathrm{C}$ and $100^{\circ} \mathrm{C}$. FoM for polysiloxane scintillator is lower with respect to EJ-299 at all the temperatures, but it increases from RT to $70^{\circ}$, where it becomes comparable with EJ-299, and then decreases.

\section{Discussion}

The lowering of the scintillation performance of polymeric scintillators with the temperature is typically attributed to temperature quenching of the dye luminescence yield and to degradation of the organic components. Here, we deepened the mechanisms underlying the deterioration with the temperature of the scintillation yield in polysiloxane based scintillators following two approaches. In fact, besides the analysis of both scintillation yield and PSD performance of the samples during heating, we studied the behaviour of samples containing only PPO (primary dye) heated in air at different temperatures.

Therefore, luminescence and weight loss measurements evidenced that the heat treatment essentially leads to release of PPO from the surface. This phenomenon can be indicated as the main scintillation degradation cause after prolonged exposure to high temperatures. In fact, we have to point out that in this work scintillation yield measurements have been performed after PSD analyses lasting several hours. During this time, the PPO diffuses out of the surface of the samples leading to the observed changes of performance.

Different processes can take place during heating: (i) diffusion of PPO through the polymer matrix favoured by the low melting point $\left(72{ }^{\circ} \mathrm{C}\right)$ of this species [36]; (ii) release of PPO from the sample surface by evaporation, as demonstrated by the measured weight loss (Fig. 2a); (iii) degradation of PPO induced by heat and environment exposure resulting in the formation of non-fluorescent, UV-Vis absorbing brown coloured by-products $[37,38]$.

The process (i) is strongly affected by the free volume of the polymer matrix, and it is therefore expected to occur more 
Table 2 Parameters obtained from the interpolation of the decay profiles in Fig. 5

\begin{tabular}{lllllllll}
\hline Sample & $\mathrm{T}\left({ }^{\circ} \mathrm{C}\right)$ & $\tau_{\text {fast }}(\mathrm{ns})$ & $\tau_{\text {slow }}(\mathrm{ns})$ & $\mathrm{A}_{\text {fast }}$ & $\mathrm{A}_{\text {slow }}$ & $\mathrm{f}_{\text {fast }}$ & $\mathrm{f}_{\text {slow }}$ & $\mathrm{f}_{\text {fast }} / \mathrm{f}_{\text {slow }}$ \\
\hline PMPS_PPO2 & 60 & $2.48 \pm 0.02$ & $25 \pm 7$ & $0.993 \pm 0.002$ & $0.007 \pm 0.002$ & $0.93 \pm 0.02$ & $0.07 \pm 0.02$ & $14 \pm 5$ \\
& 100 & $2.13 \pm 0.01$ & $25 \pm 3$ & $0.991 \pm 0.001$ & $0.009 \pm 0.001$ & $0.90 \pm 0.01$ & $0.10 \pm 0.01$ & $9 \pm 1$ \\
& 150 & $2.22 \pm 0.01$ & $25 \pm 8$ & $0.997 \pm 0.001$ & $0.003 \pm 0.001$ & $0.97 \pm 0.02$ & $0.03 \pm 0.02$ & $30 \pm 14$ \\
PMPS_PPO6 & 60 & $2.01 \pm 0.01$ & $14.1 \pm 0.2$ & $0.876 \pm 0.002$ & $0.124 \pm 0.002$ & $0.502 \pm 0.005$ & $0.496 \pm 0.005$ & $1.01 \pm 0.02$ \\
& 100 & $2.03 \pm 0.01$ & $14.4 \pm 0.2$ & $0.907 \pm 0.002$ & $0.093 \pm 0.002$ & $0.579 \pm 0.007$ & $0.421 \pm 0.007$ & $1.38 \pm 0.04$ \\
& 150 & $2.02 \pm 0.01$ & $17.8 \pm 0.5$ & $0.960 \pm 0.001$ & $0.040 \pm 0.001$ & $0.731 \pm 0.007$ & $0.269 \pm 0.007$ & $2.72 \pm 0.10$ \\
\hline
\end{tabular}
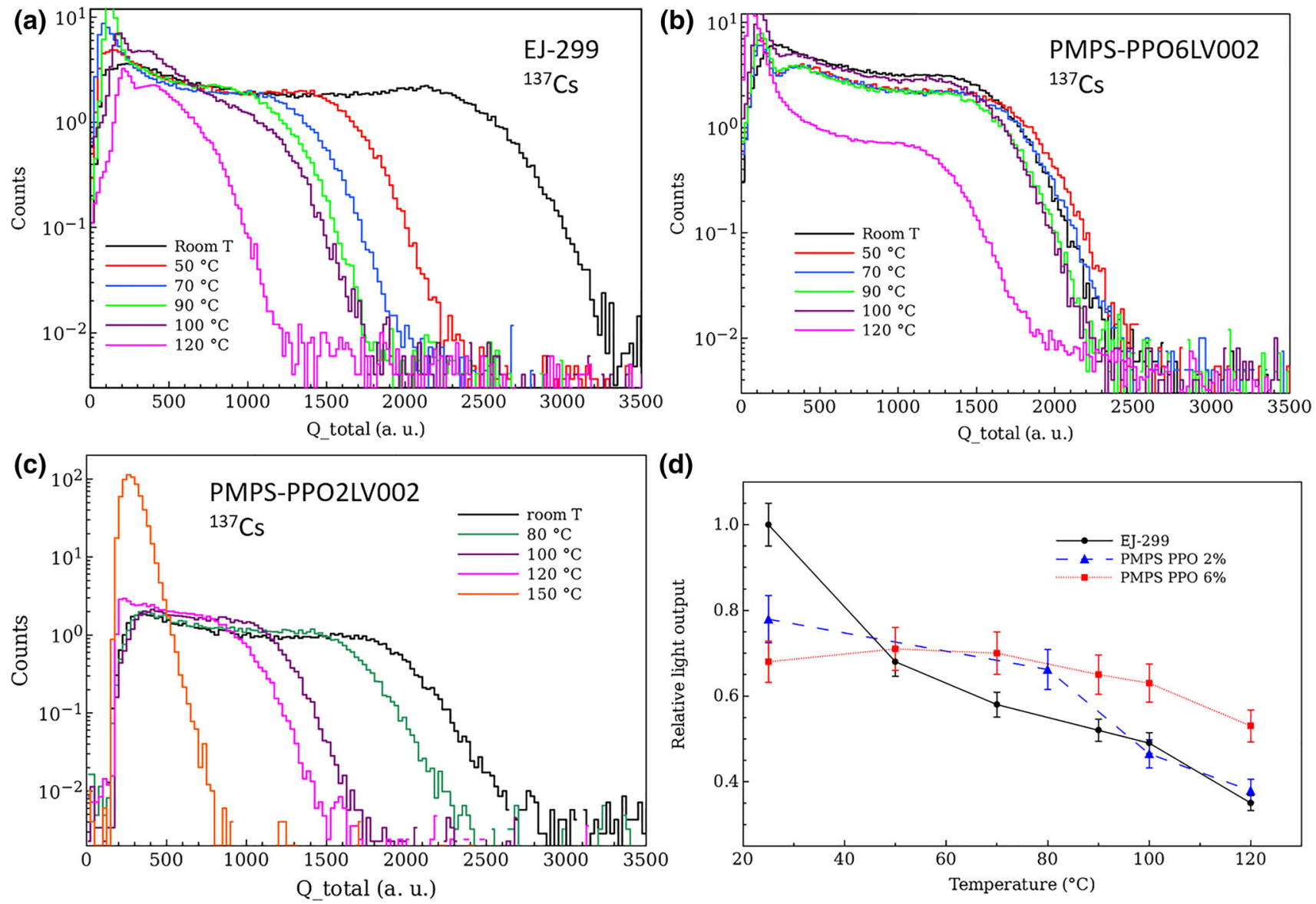

Fig. 6 Pulse height spectra collected by exposing the standard EJ-299 (a) and the siloxane-based scintillator with 6\% PPO (b) and 2\% PPO (c) to $\gamma$-rays from ${ }^{137} \mathrm{Cs}$ source. The $\mathrm{LY}$ as relative \% of EJ-299 versus

temperature is reported in the graph (d). All the measurements have been collected with the light guide coupled to the scintillator, as shown in the scheme of Fig. 1a

favourably in polysiloxanes than in PVT, owing to the high free volume characteristic of PMPS induced by the mobility of siloxane macromolecules [39]. As a matter of fact, this is supported by diffusion data reported in Fig. 2b, clearly pointing to a much higher PPO mobility in the PMPS matrix than in rigid PVT.

Hence, the remarkable decrease of scintillation light output for $2 \%$ PPO samples may be related mainly to the desorption of PPO from the sample surface evidenced by weight loss. The hypothesis that light output decrease is caused by dye molecule degradation due to thermally induced oxidation (process iii), as evidenced in oxazole and derivatives [37] and recently observed in liquid scintillators [38], can be excluded for this concentration. In fact, transmittance spectra and optical clarity are almost unchanged after thermal annealing up to $150{ }^{\circ} \mathrm{C}$ (Fig. 3c).

On the other hand, in samples with $6 \%$ PPO, the scintillation efficiency does not decrease up to $100{ }^{\circ} \mathrm{C}$. It can 

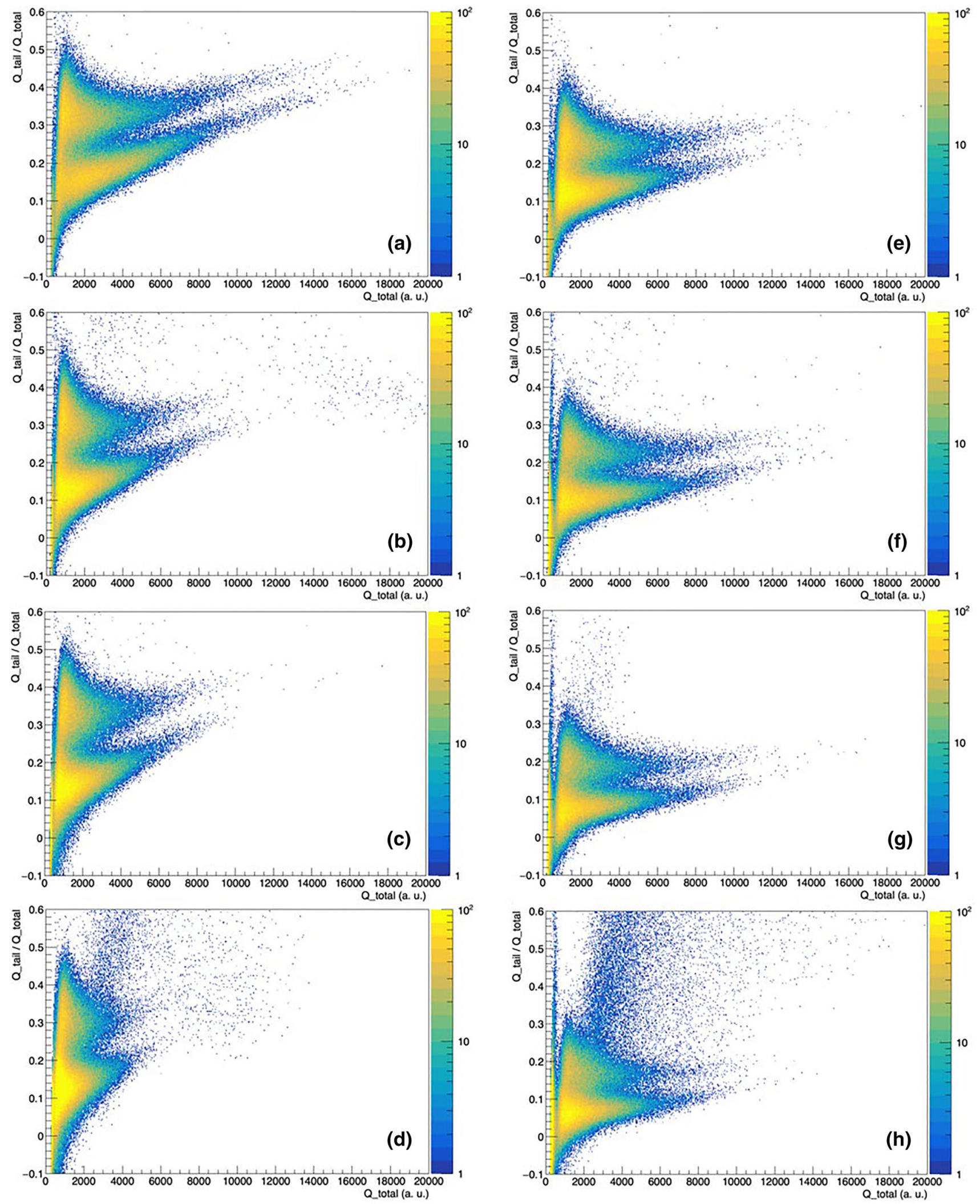

Fig. 7 2D plots (pulse shape discrimination parameter versus total integral) corresponding to the standard EJ-299 at a room temperature, $\mathbf{b} 70{ }^{\circ} \mathrm{C}$, c $100{ }^{\circ} \mathrm{C}, \mathbf{d ~} 120^{\circ} \mathrm{C}$, and PMPS based scintillator with $6 \% \mathrm{PPO}$ at e room temperature, $\mathbf{f} 70^{\circ} \mathrm{C}, \mathbf{g ~} 100{ }^{\circ} \mathrm{C}, \mathbf{h ~} 120^{\circ} \mathrm{C}$ 
(a)

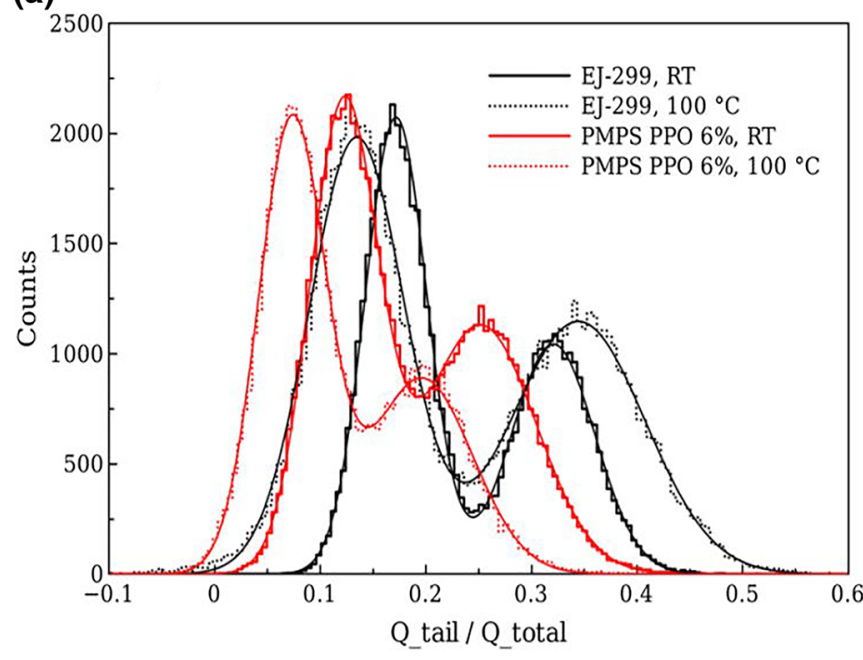

Fig. 8 a $Q_{\text {tail }} / Q_{\text {total }}$ histograms corresponding to the EJ-299 and to the PMPS scintillators, at room temperature and $100{ }^{\circ} \mathrm{C}$. Only events with light output in the range $(480 \pm 75)$ keVee were included. Gaussian (b)

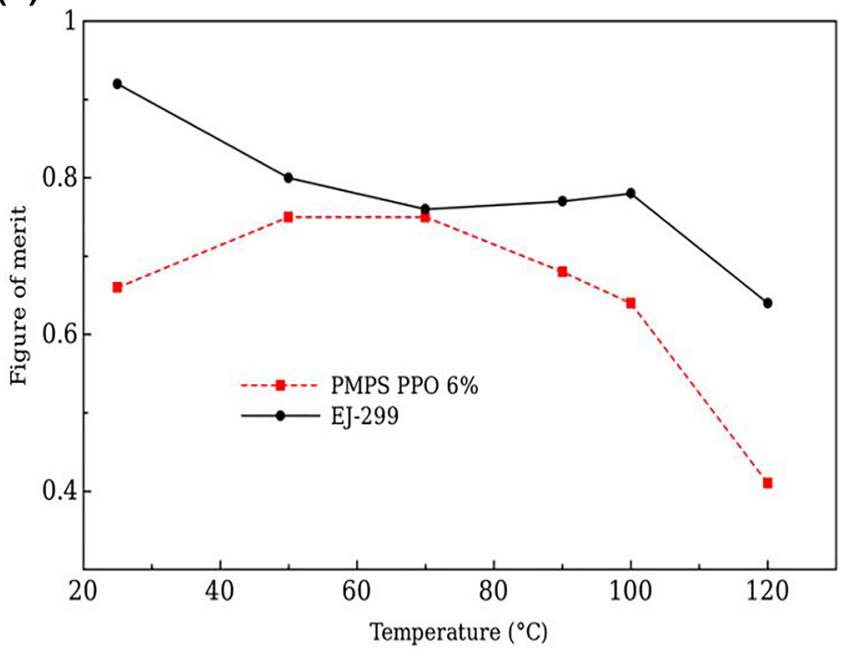

fits are shown by the continuous lines. b Figure of merit as a function of the scintillator annealing temperature (estimated statistical error \pm 0.01 for each value)

absorption centres, i.e. the inner filter effect. In this work, we can infer that the same mechanism is possible, although the matrix is solid glassy PVT instead of liquid linear alkyl benzene (LAB). Moreover, in EJ-299 PPO concentration is quite high [16] and a large number of oxidized molecules can be the cause of a so intense yellow colour inducing the loss of optical clarity and, consequently, of scintillation yield (Fig. 6d). Together with the yellowing due to the thermal degradation of PPO, in EJ-299 the PVT softening can also contribute to the degradation of light output, enabling non-radiative decay pathways for the dye molecules. In fact, PVT is characterized by a softening point around $70-90{ }^{\circ} \mathrm{C}$, which is lower than most of the inspected temperatures. On the other hand, the high loss of PPO, reported in Table 1 and Fig. 2a, cannot be indicated as the main cause of the lowering of the scintillation yield, since the dye concentration is so high that the total amount left should be enough for preserving the scintillation properties.

Concerning the FoM for $n / \gamma$ discrimination, it is not severely jeopardized up to $70{ }^{\circ} \mathrm{C}$ for PMPS sample, suffering an important worsening at higher temperatures (Fig. 7). It is worth to mention that the diffusion coefficient of PPO in PMPS reported in Fig. $2 b$ varies of three orders of magnitude as the temperature rises from 100 to $150{ }^{\circ} \mathrm{C}$, thus proving a relevant thermal effect on the mobility of PPO through the siloxane macromolecules. Therefore, it can be envisaged that, since PSD is promoted by the proximity of dye molecules [31], the mobility enhancement induced by the annealing can actually help the PSD process, up to a certain limit, beyond which the impoverishment of PPO due to out-diffusion causes dissociation of excimers and PSD 
efficiency loss, as evidenced by the steep In the case of EJ299, where the initial amount of PPO is much higher, the worsening of PSD performances, although moderate, occurs already at low annealing temperature $\left(70^{\circ} \mathrm{C}\right)$, following the light yield loss trend. Therefore, in this case the same motivations described above for light output decrease account for PSD performance reduction, i.e. PPO thermal degradation and non-radiative decay due to enhanced chain mobility.

The oblique stripe on 2D plots, particularly evident in Fig. 7f, g, may be related to the real time evolution of PPO distribution, since the overall analysis took 20-24 hours. A similar, though much less intense, stripe-shaped signal appears for EJ-299 as well, but only for sample treated at $120{ }^{\circ} \mathrm{C}$ (Fig. 7d), thus supporting the fact that in PVT matrix mobility and diffusion of PPO is hampered with respect to polysiloxane and it occurs at a sensible extent at high temperature only.

\section{Conclusions}

This work reports the effects on light yield and PSD features caused by prolonged heating at temperatures as high as $150{ }^{\circ} \mathrm{C}$ of polysiloxane based scintillators, as compared to the PVT based EJ-299 scintillator. In particular, the study of the PPO as primary dye dispersed in the polymer networks evidenced for the first time how the temperature effects are related to the enhanced mobility and out-diffusion from the scintillators of the dye molecule. Up to $120^{\circ} \mathrm{C}$, it was observed an evident loss of dye, leading to a decrease of the scintillation yield in low concentration polysiloxane samples. At higher concentration, the scintillation yield slightly increases, and it is stable up to $100{ }^{\circ} \mathrm{C}$ due to the lowering of concentration quenching effects. To this regard, up to 120 ${ }^{\circ} \mathrm{C}$ temperature effects on polysiloxane based samples can be attributed only to the PPO mobility and out-diffusion. On the other hand, PVT based samples with high PPO concentrations present an evident scintillation yield lowering due to PPO thermal degradation and formation of absorbing colour centres, accompanied by activation of non-radiative decay channels through polymer chain mobility. PSD performances are less affected by the temperature in the PMPS sample up to $100{ }^{\circ} \mathrm{C}$, where diffusion through the polysiloxane is favoured with respect to polyvinyltoluene matrix of EJ-299, hence the aggregation of PPO, and in turn excimers formation, is promoted. This process positively affects PSD up to a certain temperature limit, above which the dye loss reaches a critical extent and excimers dissociation with consequent deterioration of PSD is observed. In EJ-299 the FoM characterizing PSD clearly decreases also at low temperature and in this case the trend is related to light yield loss due to inner filter effect caused by thermal degradation of PPO, which is present in high concentration.
The demonstrated feasibility of the discrimination between fast neutrons and $\gamma$-rays at temperatures as high as $100^{\circ} \mathrm{C}$ for prolonged times, using siloxane-based scintillators, clearly widens the range of application of organic-based scintillators particularly at the high temperature induced by high irradiation fluxes. Further work is in progress to synthesize a polysiloxane with a high molar concentration of phenyl groups to preserve dyes solubility, but with higher cross-link density: this might inhibit PPO out-diffusion and allow the use of polysiloxane based scintillators at an even higher temperature.

Acknowledgements This work has been funded by the University of Padova, Department of Physics and Astronomy under the Research Grant assigned to Felix Pino, and by the $\mathrm{V}^{\text {th }}$ Commission of INFN, under the research project "FIRE" and the Research Grant assigned to Rosasilvia Raggio.

Data Availability Statement This manuscript has no associated data or the data will not be deposited. [Authors' comment: All the useful data have been presented in the manuscript, in case of need, the authors may provide raw data files used to build-up plots and graphs.]

Open Access This article is licensed under a Creative Commons Attribution 4.0 International License, which permits use, sharing, adaptation, distribution and reproduction in any medium or format, as long as you give appropriate credit to the original author(s) and the source, provide a link to the Creative Commons licence, and indicate if changes were made. The images or other third party material in this article are included in the article's Creative Commons licence, unless indicated otherwise in a credit line to the material. If material is not included in the article's Creative Commons licence and your intended use is not permitted by statutory regulation or exceeds the permitted use, you will need to obtain permission directly from the copyright holder. To view a copy of this licence, visit http://creativecomm ons.org/licenses/by/4.0/.

Funded by $\mathrm{SCOAP}^{3}$.

\section{References}

1. H. Jivan, B. Mellado, E. Sideras-Haddad, R. Erasmus, S. Liao, M. Madhuku, G. Peters, O. Solvyanov, J. Phys. Conf. Ser. 623, 012016 (2015)

2. M. Pari, G. Ballerini, A. Berra, R. Boanta, M. Bonesini, C. Brizzolari, G. Brunetti, M. Calviani, S. Carturan, M.G. Catanesi, S. Cecchini, A. Coffani, F. Cindolo, G. Collazuol, E. Conti, F. Dal Corso, G. De Rosa, C. Delogu, A. Gola, R. A. Intonti, C. Jollet, Y. Kudenko, M. Laveder, A. Longhin, P.F. Loverre, L. Ludovici, L. Magaletti, G. Mandrioli, A. Margotti, V. Mascagna, N. Mauri, A. Meregaglia, M. Mezzetto, M. Nessi, A. Paoloni, E. Parozzi, L. Pasqualini, G. Paternoster, L. Patrizii, C. Piemonte, M. Pozzato, F. Pupilli, M. Prest, E. Radicioni, C. Riccio, A.C. Ruggeri, G. Sirri, M. Soldani, M. Tenti, M. Torti, F. Terranova, E. Vallazza, M. Vesco, L. Votano, Nucl. Instruments Methods Phys. Res. Sect. A Accel. Spectrometers, Detect. Assoc. Equip. 936, 148 (2019)

3. R.T. Kouzes, E.R. Siciliano, J.H. Ely, P.E. Keller, R.J. McConn, Nucl. Instruments Methods Phys. Res. Sect. A Accel. Spectrometers, Detect. Assoc. Equip. 584, 383 (2008)

4. J.W. Bae, H.R. Kim, Nucl. Eng. Technol. 52, 1259 (2020)

5. Mirion Technologies, (n.d.)

6. C.W.E. Van Eijk, Radiat. Meas. 38, 337 (2004) 
7. J. Harmon, J. Gaynor, V. Feygelman, J. Walker, Nucl. Instruments Methods Phys. Res. Sect. B Beam Interact. with Mater. Atoms 53, 309 (1991)

8. A. Quaranta, S. M. Carturan, T. Marchi, V. L. Kravchuk, F. Gramegna, G. Maggioni, M. Degerlier, IEEE Trans. Nucl. Sci. 57, (2010)

9. S. M. Carturan, T. Marchi, E. Fanchini, R. De Vita, P. Finocchiaro, A. Pappalardo, Eur. Phys. J. Plus 129, (2014)

10. A. Quaranta, S. Carturan, M. Cinausero, T. Marchi, F. Gramegna, M. Degerlier, A. Cemmi, S. Baccaro, Mater. Chem. Phys. 137, (2013)

11. S. Carturan, A. Quaranta, T. Marchi, F. Gramegna, M. Degerlier, M. Cinausero, V. L. Kravchuk, M. Poggi, Radiat. Prot. Dosimetry 143, (2011)

12. S.M. Carturan, T. Marchi, G. Maggioni, F. Gramegna, M. Degerlier, M. Cinausero, M.D. Palma, A. Quaranta, J. Phys. Conf. Ser. 620, 012010 (2015)

13. S.M. Carturan, M. Vesco, I. Bonesso, A. Quaranta, G. Maggioni, L. Stevanato, E. Zanazzi, T. Marchi, D. Fabris, M. Cinausero, F. Pino, F. Gramegna, Nucl. Instruments Methods Phys. Res. Sect. A Accel. Spectrometers, Detect. Assoc. Equip. 925, 109 (2019)

14. T. Marchi, F. Pino, C.L. Fontana, A. Quaranta, E. Zanazzi, M. Vesco, M. Cinausero, N. Daldosso, V. Paterlini, F. Gramegna, S. Moretto, G. Collazuol, M. Degerlier, D. Fabris, S.M. Carturan, Sci. Rep. 9, 9154 (2019)

15. Eljen Technology, https://eljentechnology.com/products/ plastic-scintillators/ej-276

16. N. Zaitseva, B.L. Rupert, I. Pawełczak, A. Glenn, H.P. Martinez, L. Carman, M. Faust, N. Cherepy, S. Payne, Nucl. Instruments Methods Phys. Res. Sect. A Accel. Spectrometers, Detect. Assoc. Equip. 668, 88 (2012)

17. G.H.V. Bertrand, M. Hamel, S. Normand, F. Sguerra, Nucl. Inst. Methods Phys. Res. A 776, 114 (2015)

18. A. Mahl, A. Lim, J. Latta, H.A. Yemam, U. Greife, A. Sellinger, Nucl. Instruments Methods Phys. Res. Sect. A Accel. Spectrometers, Detect. Assoc. Equip. 884, 113 (2018)

19. A. Lim, G. Hernandez, J. Latta, H.A. Yemam, W. Senevirathna, U. Greife, A. Sellinger, A.C.S. Appl, Polym. Mater. 1, 1420 (2019)

20. G. Barbarino, M. Boscherini, D. Campana, W. Filrnkranz, W. Menn, M. Orazi, G. Osteria, M. Simon, E. Webera, Nucl. Phys. B - Proc. Suppl. 125, 298 (2003)
21. N.P. Zaitseva, A.M. Glenn, A.N. Mabe, M.L. Carman, C.R. Hurlbut, J.W. Inman, S.A. Payne, Nucl. Instruments Methods Phys. Res. Sect. A Accel. Spectrometers, Detect. Assoc. Equip. 889, 97 (2018)

22. A. Lim, J. Arrue, P.B. Rose, A. Sellinger, A.S. Erickson, ACS Appl. Polym. Mater. 2, 3657 (2020)

23. O. Çoban, Appl. Surf. Sci. 317, 405 (2014)

24. C.L. Fontana, https://gitlab.com/cristiano.fontana/abcd

25. C.L. Fontana, A. Carnera, M. Lunardon, F.E. Pino, C. Sada, F. Soramel, L. Stevanato, S. Moretto, Int. J. Mod. Phys. Conf. Ser. 48, 1860118 (2018)

26. J. Cranck, The Mathematics of Diffusion, 2nd edn. (Bristol, UK, 1975)

27. M. Lay, M.R. Ramli, R. Ramli, N.C. Mang, Z. Ahmad, J. Appl. Polym. Sci. 136, 2 (2019)

28. S.L. Malhotra, J. Hesse, L.-P. Blanchard, Polymer (Guildf). 16, 81 (1975)

29. I.B. Berlman, J. Chem. Phys. 34, 1083 (1961)

30. J.B. Birks, L.G. Christophorou, Nature 197, 1064 (1963)

31. D.L. Horrocks, J. Chem. Phys. 51, 5443 (1969)

32. H. Lami, G. Laustriat, J. Chem. Phys. 48, 1832 (1968)

33. B. Valeur, Molecular Fluorescence (Wiley, 2001)

34. O.J. Steingraber, I.B. Berlman, Rev. Sci. Instrum. 34, 524 (1963)

35. J. Yguerabide, M. Burton, J. Chem. Phys. 37, 1757 (1962)

36. H.A. Yemam, A. Mahl, J.S. Tinkham, J.T. Koubek, U. Greife, A. Sellinger, Chem. - A Eur. J. 23, 8921 (2017)

37. M.V. George, V. Bhat, Chem. Rev. 79, 447 (1979)

38. N.L.P. Andrews, J.Z. Fan, R.L. Forward, M.C. Chen, H.-P. Loock, Phys. Chem. Chem. Phys. 19, 73 (2017)

39. S.A. Stern, V.M. Shah, B.J. Hardy, 25, (1987) 